\title{
Early short-term ivabradine treatment in new-onset acute systolic heart failure and sinus tachycardia patients with inflammatory rheumatic disease
}

\author{
WEI WU ${ }^{1}$, LIXI ZHANG ${ }^{1}$, JIULIANG ZHAO ${ }^{2}$, YUCHAO GUO $^{3}$, JINJING LIU $^{2}$, DI SHI ${ }^{4}$, \\ JING YANG ${ }^{4}$, YINGXIAN LIU ${ }^{1}$, JINZHI LAI ${ }^{1}$ and ZHUJUN SHEN ${ }^{1}$
}

\begin{abstract}
Departments of ${ }^{1}$ Cardiology and ${ }^{2}$ Rheumatology, Peking Union Medical College Hospital, Peking Union Medical College and Chinese Academy of Medical Science, Beijing 100730; ${ }^{3}$ Department of Cardiology, The Second Affiliated Hospital of Zhejiang University School of Medicine, Hangzhou, Zhejiang 310009; ${ }^{4}$ Department of Emergency, Peking Union Medical College Hospital, Peking Union Medical College and Chinese Academy of Medical Science, Beijing, P.R. China
\end{abstract}

Received September 1, 2018; Accepted April 5, 2019

DOI: $10.3892 /$ etm.2019.7531

\begin{abstract}
Acute heart failure (AHF) is a common complication of inflammatory rheumatic disease (IRD) and usually coexists with tachycardia. Ivabradine, a direct sinus node inhibitor, which was proven to have favorable effects in patients with chronic HF (CHF), has not been sufficiently evaluated in AHF patients regarding its efficacy and safety. The present study sought to explore the effectiveness of early short-term ivabradine treatment in new-onset AHF and concurrent sinus tachycardia in patients with IRD. A total of 12 consecutive patients with IRD, who had new-onset AHF and concurrent sinus tachycardia, were prescribed ivabradine and were retrospectively recruited. Standard medication therapy for AHF was also administered. The heart rate (HR), left ventricular ejection fraction (LVEF), biomarkers of HF and New York Heart Association (NYHA) classification score were compared prior to and after ivabradine treatment. After $48 \mathrm{~h}$ of treatment with ivabradine, the mean resting HR decreased from $118.0 \pm 13.8$ to $83.3 \pm 7.3 \mathrm{bpm}(\mathrm{P}<0.001)$. Transthoracic echocardiography indicated a significant improvement in the LVEF on an average of 2 weeks after ivabradine prescription when compared with the baseline evaluation $(51.2 \pm 8.4$ vs. $38.0 \pm 9.0 \%$; $\mathrm{P}<0.001)$. In addition, ivabradine treatment resulted in significantly decreased $\mathrm{N}$-terminal proB-type natriuretic peptide $(4,900 \pm 3,672$ vs. $16,806 \pm 16,130 \mathrm{pg} / \mathrm{ml} ; \mathrm{P}=0.045)$ and improvement of the NYHA classification score $(2.3 \pm 0.6$ vs. $3.5 \pm 0.5 ; \mathrm{P}<0.001)$ at 2 weeks when compared with the baseline. Overall, the results of the present study suggested that early use of ivabradine is
\end{abstract}

Correspondence to: Dr Zhujun Shen, Department of Cardiology, Peking Union Medical College Hospital, Peking Union Medical College and Chinese Academy of Medical Science, 1 Shuai Fu Yuan, Beijing 100730, P.R. China

E-mail: zhujun66shen@126.com

Key words: acute heart failure, inflammatory rheumatic disease, ivabradine, heart rate, tachycardiomyopathy safe in IRD patients with new-onset AHF and enhances the sinus rate reduction, which may improve heart function.

\section{Introduction}

The heart is an important target organ involved in inflammatory rheumatic diseases (IRD) and cardiovascular death is one of the most frequent causes of mortality among patients with $\operatorname{IRD}(1,2)$. Heart involvement may occur during the acute phase of IRD, including myocarditis or coronary artery macro- or micro-vasculitis, which probably leads to acute heart failure (AHF) (3). On the other hand, infection, anemia, tachycardia, renal dysfunction, anxiety, glucocorticoid usage and other IRD-associated clinical conditions may increase the heart rate (HR) and exacerbate AHF among these patients. In either of these two scenarios, a high incidence of cardiac arrhythmias has been reported and sinus tachycardia is one of the major manifestations in IRD patients (4).

Although an increase in HR may be a compensatory response to reduced left ventricular (LV) stroke volume and cardiac output in the setting of AHF, it may also deteriorate LV systolic function by causing tachycardiomyopathy (TCM) (5). At present, TCM is mainly defined by the following clinical criteria: Sustained HR >100 bpm, exclusion of other causes of $\mathrm{HF}$, and partial or complete recovery of LV function after restoration of the sinus rhythm or rate control $(6,7)$. Accordingly, it may be implied that HR control, in addition to the application of diuretics and/or inotropes, is crucial for the treatment of decompensated AHF patients without any previously diagnosed heart disease. Low doses of $\beta$-blockers are usually applied for the initial treatment of tachycardia in IRD patients with systolic HF. Subsequently, the dose is slowly titrated to tolerable levels to achieve the target HR. This process may take up to 2-3 months. As a selective sinus node I(f) current inhibitor, ivabradine significantly prolongs the diastolic phase, effectively reduces the HR $(8,9)$ and subsequently improves the prognosis of patients with chronic HF (10). However, clinical evidence to support the application of ivabradine in patients with acute systolic HF is currently limited (11). Therefore, the 
present study aimed to investigate the safety and potential efficacy of early and short-term use of ivabradine in IRD patients with sinus tachycardia and new-onset AHF.

\section{Patients and methods}

Patient population. The present study was a retrospective observational study, in which IRD patients who experienced new-onset acute systolic $\mathrm{HF}$ as well as sinus tachycardia and received ivabradine therapy in Peking Union Medical College Hospital (Beijing, China) between February 2017 and May 2017 were enrolled. All patients received ivabradine for HR control in addition to the standard treatment for AHF, including loop diuretics and/or mechanical ventilation when required. Selection and dosages of other medications, including $\beta$-blockers, were determined by individual clinical settings.

The inclusion criteria were as follows: i) New-onset AHF; ii) LV ejection fraction (EF) $<50 \%$ determined by transthoracic echocardiography (Simpson's method); iii) sinus tachycardia with HR >100 bpm; and iv) unequivocal diagnosis of IRD prior to or concurrent with presentation of the new-onset AHF. New-onset AHF was defined as a diagnosis of AHF with presence of pulmonary edema for the first time with a reduction in EF by $<50 \%$ but without evidence of history of $\mathrm{HF}$ in the previous 3 months. The exclusion criteria included the following: i) Previously diagnosed or documented HF in the 3 months before the study; ii) other heart rhythm indicated by in-hospital electrocardiogram (e.g., supraventricular, atrial, ventricular); iii) cardiac shock; iv) acute coronary syndrome; v) previous treatment with ivabradine; and vi) hemodynamically significant valvular disease. All patients had systolic blood pressures of $>90 \mathrm{mmHg}$ without inotrope/vasopressor usage.

A total of 156 patients with IRD were admitted to our hospital during the enrolment period, of which 14 patients (9.0\%) met the eligibility criteria. A total of 12 patients (10 females and 2 males; age range, 16-63 years; mean age, $33.8 \pm 12.5$ years) provided written informed consent and received subsequent ivabradine treatment, while the other two patients refused. All patients or their legal guardians provided written informed consent for administration of ivabradine, since this medication had not been indicated for treating AHF clinically. The present study complied with the ethical guidelines of the 1964 Declaration of Helsinki and its revision from 2002. The study protocol was reviewed and approved by the ethics committee of Peking Union Medical College Hospital (Beijing, China).

Medication. A special group of cardiologists were responsible for the patients' medications for HF, including the administration, dosing and timing of ivabradine. Classic medical treatment for AHF, including diuretics, oxygen therapy and fluid restrictions, were selected according to the patients' clinical situations. Application of $\beta$-adrenergic receptor blockers was selected according to the clinicians' preference. Most patients received no or moderate dosages of $\beta$-blockers as initial therapy for AHF. Use or discontinuation of glucocorticoids and other immunosuppressant drugs for IRD were determined by clinical rheumatologists independent of AHF therapy.
When HR reduction was considered necessary by the cardiologists and it was not possible to rapidly titrate $\beta$-blockers for this purpose, ivabradine administration was selected for these AHF patients according to the inclusion and exclusion criteria. Due to the uncertainty of efficacy and safety of ivabradine treatment in decompensated AHF patients, most patients were given a low dosage of $2.5 \mathrm{mg}$ twice daily, and not $5 \mathrm{mg}$ twice daily as applied in the initial therapy for chronic HF patients (10). If a target HR of $80 \mathrm{bpm}$ was not achieved within the first three days of treatment, the dosage was increased to $5 \mathrm{mg}$ twice daily. The application or dose increase of a $\beta$-blocker in combination with ivabradine depended on the clinician's judgement and/or the patient's general condition. If the patient's LVEF during the follow-up echocardiogram exceeded $50 \%$, the ivabradine dosage was not further increased, because the HR would then controllable by a rapid increase in the $\beta$-blocker dose.

Observational indexes. The primary outcome was the improvement of the EF, which was measured using the biplane Simpson's method on transthoracic echocardiography. Initial echocardiographic evaluation was performed within 3 days prior to initiation of ivabradine treatment, and the follow-up evaluation was performed $\sim 2$ weeks after the first ivabradine administration. The secondary endpoints included HR changes at $48 \mathrm{~h}$ after the first ivabradine administration vs. baseline, changes in B-type natriuretic peptide (BNP)/N-terminal proBNP (NT-proBNP) levels and cardiac function according to the New York Heart Association (NYHA) classification of $\mathrm{HF}$ at 2 weeks after the first ivabradine use vs. baseline. Furthermore, blood pressure, liver and renal function, and plasma lactic acid changes prior to and after ivabradine use were repeatedly measured for safety assessment.

Statistical analysis. All analyses were performed using SPSS 19.0 statistical software (IBM Corp.). Values are expressed as the mean \pm standard deviation. The Kolmogorov-Smirnov test was used to evaluate whether the distributions of continuous variables were normal. Comparisons of LVEF, HR and BNP/NT-proBNP prior to and after ivabradine administration were performed using paired t-tests. Comparisons of the NYHA function classification prior to and after ivabradine administration were performed using the Wilcoxon signed-rank test, which is a paired non-parametric test. $\mathrm{P}<0.05$ was considered to indicate statistical significance.

\section{Results}

Patient characteristics. The basic clinical characteristics of the patients are presented in Table I. Concomitant IRDs included systemic lupus erythematosus (SLE), secondary antiphospholipid syndrome (APS), granulomatosis with polyangiitis (GPA) and polymyositis/dermatomyositis (PM/DM).

$H R$ reduction. The patients of the present study presented with a significant HR reduction at $48 \mathrm{~h}$ after ivabradine treatment (Fig. 1), and the mean resting HR was decreased from $118.0 \pm 13.8$ to $83.3 \pm 7.3 \mathrm{bpm}(\mathrm{P}<0.001)$. Fig. 2 provides the changes in HR and blood pressure in a typical IRD patient (case no. 3; female; age, 17 years), who received mechanical 


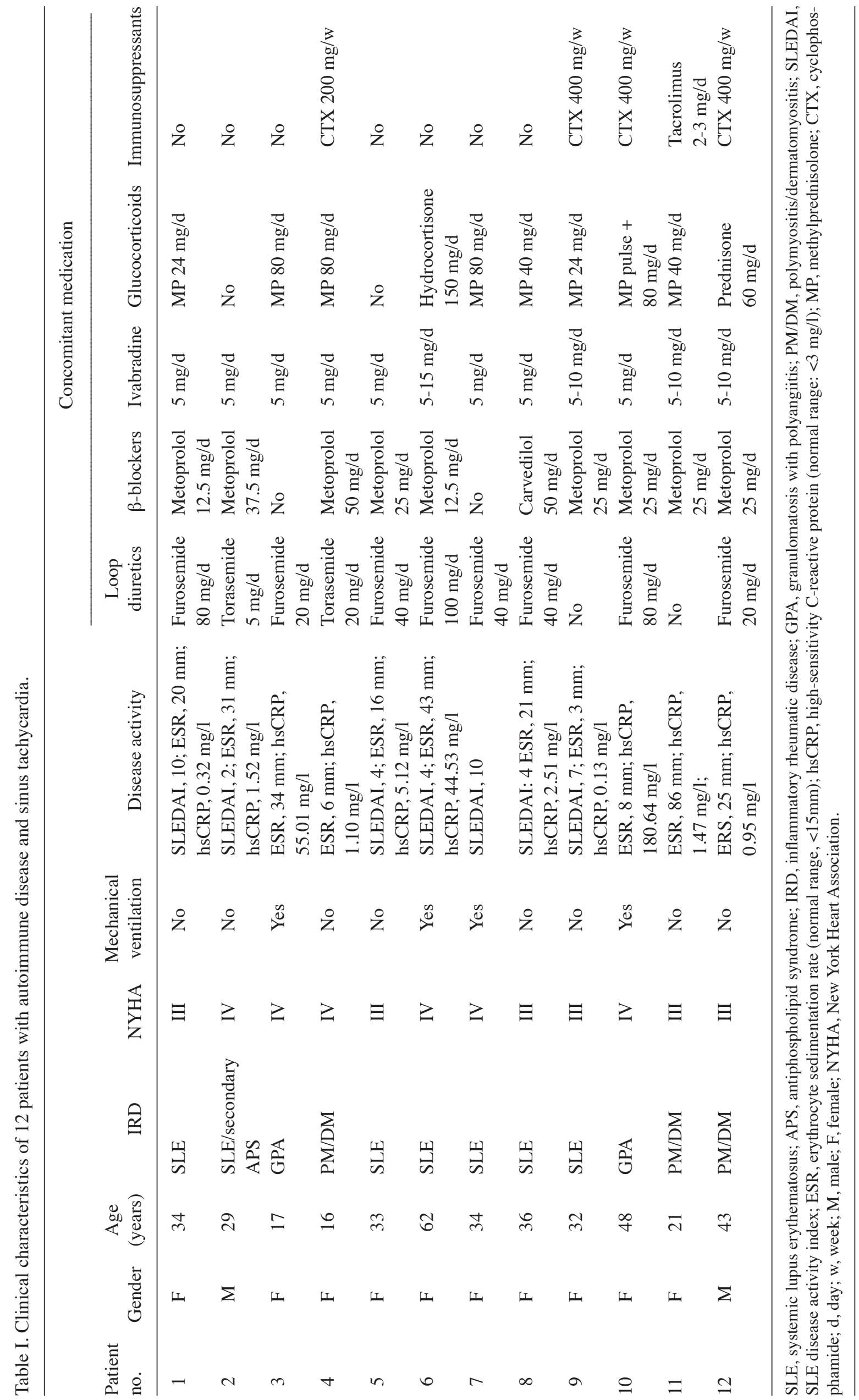




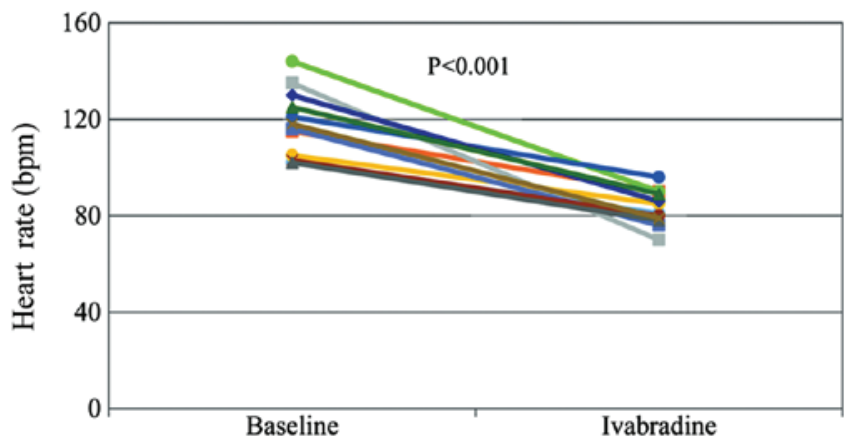

Figure 1. Changes in heart rate prior to and $48 \mathrm{~h}$ after ivabradine treatment for each of the 12 patients.

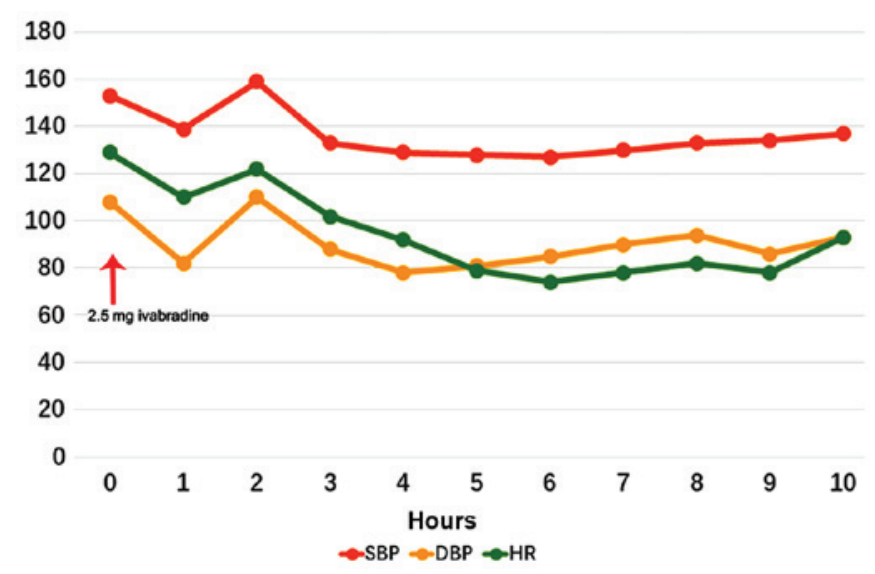

Figure 2. SBP, DBP and HR in Patient 3 at each hour after $2.5 \mathrm{mg}$ ivabradine administration. SBP, systolic blood pressure; DBP, diastolic blood pressure; HR, heart rate.

ventilation due to pulmonary edema and hypoxemia caused by AHF, over the first $10 \mathrm{~h}$. Within $6 \mathrm{~h}$ of $2.5 \mathrm{mg}$ ivabradine administration, her HR dropped from 129 to $74 \mathrm{bpm}$ without significant changes in blood pressure. Simultaneously, her LVEF increased from 28 to $40 \%$. There were no dosage modifications of ivabradine or new prescriptions of $\beta$-blockers within the first $48 \mathrm{~h}$ for all patients, and the dosage of sedatives was not modified if the patients were intubated. If the patient's HR persistently remained $>80 \mathrm{bpm}$, the ivabradine dose was titrated to $10 \mathrm{mg} /$ day after 3 days or even to $15 \mathrm{mg} / \mathrm{day}$ thereafter.

Transthoracic echocardiography changes. The interval between the first dose of ivabradine and echocardiography re-evaluation ranged from 10 to 20 days (mean, $14.0 \pm 2.6$ days). A significant improvement in LVEF was seen after ivabradine treatment ( $38.0 \pm 9.0$ vs. $51.2 \pm 8.4 \%$; P $<0.001$; Fig. 3$)$. Of the 12 patients, 8 only received $5 \mathrm{mg}$ /day of ivabradine and the HR was $<80 \mathrm{bpm}$ at the time of echocardiography re-evaluation. Another 3 patients received $10 \mathrm{mg} /$ day, and 1 patient required $15 \mathrm{mg} / \mathrm{day}$ of ivabradine to reach the target HR. After the increase in $\mathrm{EF}$ was recognized, the $\beta$-blocker dose was rapidly increased to reduce the HR. The average period of ivabradine administration ranged from 5 to 30 days (mean, $18.1 \pm 8.8$ days), depending on the HR control, $\beta$-blocker usage and mitigation of systolic AHF.

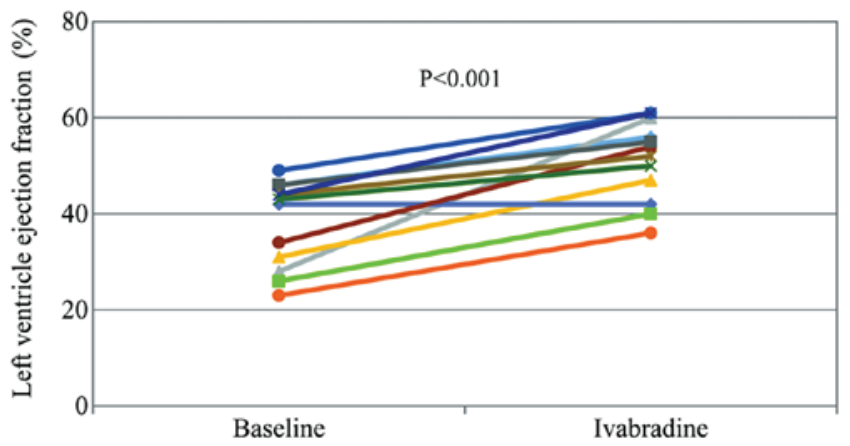

Figure 3. Left ventricle ejection fraction determined by initial and 2 week follow-up echocardiography for each of the 12 patients.

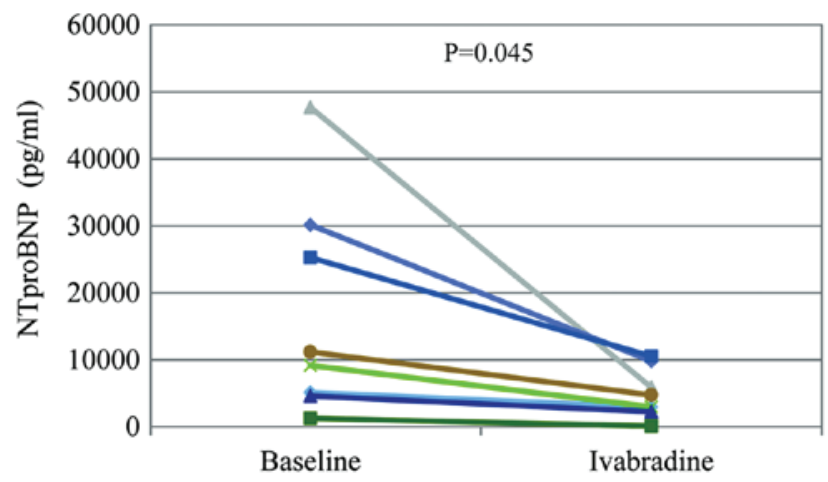

Figure 4. NT-proBNP levels at the initial and 2 week follow-up examination $(\mathrm{n}=8)$. NT-proBNP, N-terminal proB-type natriuretic peptide.

Comparison of NT-proBNP and BNP levels prior to and after treatment. NT-proBNP and BNP levels were examined at baseline and at an average of $14.2 \pm 2.7$ days (5-20 days) after the commencement of ivabradine treatment. A total of 8 patients were subjected to NT-proBNP testing and the remaining 4 were subjected to BNP testing due to their mild to severe renal dysfunction. As indicated in Fig. 4, the NT-proBNP level after ivabradine treatment was significantly lower than the initial level $(4,900 \pm 3,672$ vs. $16,806 \pm 16,130 \mathrm{pg} / \mathrm{ml} ; \mathrm{P}=0.045)$. There was no significant difference between BNP levels prior to and after treatment with ivabradine $(427 \pm 282$ vs. $318 \pm 145 \mathrm{ng} / \mathrm{l}$; $\mathrm{P}=0.528$; Table II).

Comparison of NYHA classification prior to and after ivabradine treatment. The NYHA classification of HF in the subjects was compared between baseline and 14 days after ivabradine treatment by using Wilcoxon's signed-rank test (Table II). With ivabradine treatment, a significant improvement in the NYHA function classification was achieved when compared with the initial evaluation $(2.3 \pm 0.6$ vs. $3.5 \pm 0.5$; $\mathrm{P}<0.001)$.

Safety. No incidences of hypertension, hypotension, bradycardia, atrial fibrillation were recorded in the patients of the current study. Liver and renal function also remained stable in these patients. Plasma lactic acid was not elevated prior to or following ivabradine treatment. Only one of the 12 patients reported the common ocular side effect of phosphene. 
Table II. Comparison of BNP levels and NYHA functional classification prior to and after ivabradine treatment.

\begin{tabular}{lccc}
\hline Parameter & Prior to treatment & After ivabradine & P-value \\
\hline BNP (ng/l) & $427 \pm 282$ & $318 \pm 145$ & 0.528 \\
NYHA score & $3.5 \pm 0.5$ & $2.3 \pm 0.6$ & $<0.001^{\text {a }}$ \\
\hline
\end{tabular}

${ }^{a}$ Wilcoxon signed-rank test. BNP, B-type natriuretic peptide; NYHA, New York Heart Association. BNP normal range, 0-100 ng/l.

\section{Discussion}

New-onset AHF with sinus tachycardia in IRD patients is commonly encountered in the clinic. According to the present study, ivabradine significantly decreased the HR, improved the LVEF and decreased NT-proBNP/BNP, thereby markedly improving cardiac function in the small sample of patients.

The causes of new-onset AHF in IRD have remained to be fully elucidated. Heart involvement and elevated HR are frequently observed in autoimmune diseases, including SLE (12,13), rheumatoid arthritis (14-16), GPA (17), PM/DM (18) and systemic sclerosis (19). Ischemic cardiomyopathy associated with coronary artery and microvascular diseases and/or inflammation-associated myocarditis are two major mechanisms underlying the elevated risks of AHF. The patients in the present study had no self-reported or medically recorded history of myocardial infarction. In addition, there were no acute coronary events at enrolment in these AHF patients. Evidence of severe microvascular disease or inflammation-associated myocarditis was insufficient in the present cohort due to inconvenient functional tests or high-resolution imaging performed in these critically ill patients. Two patients in the present study received gadolinium-enhanced cardiac magnetic resonance and did not to exhibit any obvious signs of myocarditis, myocardial edema or fibrosis. However, SLE myocarditis was the most characteristic feature of myocardial involvement in SLE but was of a subclinical nature $(20,21)$. No endomyocardial biopsy was performed in this group of patients.

In the present study, most cases of $\mathrm{AHF}$ were triggered by bacterial or fungal infections. Of the 12 patients, 7 received long-term regular corticosteroid and/or immunosuppressant therapy and remained stable prior to the onset of AHF. The general activity evaluations in the 7 SLE patients were mild to moderate according to the SLE Disease Activity Index score. Of note, other target organs involved in IRD were relatively 'silent', suggesting that new-onset AHF was unlikely caused by exacerbated IRD. All patients in the present study shared a common sign of inappropriate sinus tachycardia prior to the onset of AHF. Thus, it may be hypothesized that TCM is an important cause for the development of AHF in these patients. For instance, case no. 3 received methylprednisolone pulse therapy and high-dose steroid maintenance treatment due to pulmonary injury by GPA, with a LVEF of $61 \%$ at that time. Her HR constantly exceeded $120 \mathrm{bpm}$ due to repeated episodes of infection and seizures, reaching $170 \mathrm{bpm}$ at one point. Decompensated AHF with an EF of $28 \%$ occurred within 2 weeks. It remains elusive whether IRD patients may have a high incidence of TCM during a state of inflammation, but sinus tachycardia was frequent in those patients.

Marked and rapid improvement of LVEF after rate control in the patients of the current study supports the present results on TCM. Quick recovery from systolic AHF in patients was mainly attributed to rapid HR control but not the effect of glucocorticoids or other immunosuppressant drugs. In most patients, a HR reduction was achieved after 2 days of treatment, which is consistent with a previous study on ivabradine treatment in AHF patients (22). Relief of HF symptoms was reported by or observed in most patients, and this alleviation was consistent with the decreased HR shortly after ivabradine treatment. Case no. 3 exhibited a marked decrease of HR and improvement of EF within hours. This patient was successfully extubated 5 days later, and 16 days later, LV systolic function returned to normal with an $\mathrm{EF}$ of $60 \%$ on the echocardiogram.

It is worth noting that most patients presented with a marked change in HR (19.0-48.1\%) after treatment with ivabradine at a relatively low dosage (2.5 $\mathrm{mg}$ twice daily). In comparison, the HR was reduced by only $10.7-13.0 \%$ in previous large clinical trials where ivabradine was given at $5 \mathrm{mg}$ twice daily $(10,23-25)$. Two factors may be accountable for this difference. First, the average HR in previous studies, whose patients had already received maximum-tolerated doses of $\beta$-blockers, was between 70 and $90 \mathrm{bpm}$, while the initial HR of the patients in the present study was $>100 \mathrm{bpm}$. Second, previous studies included patients with stable coronary artery disease and/or CHF (10,23-25); however, only patients with acute decompensated HF were included in the present study. $\mathrm{HR}$ reduction by ivabradine led to improvement of AHF, which consequently reduced HR. Given the obvious reversibility of TCM, the HR tended to spontaneously decrease after AHF and tachycardia was successfully controlled.

The usage and dosages of $\beta$-blockers were not limited in the present study. The mean duration of ivabradine treatment was short-term ( 18.1 days). During this period, most patients had no or only one increase in the dose of $\beta$-blockers. The daily dose of $\beta$-blocker did not exceed $50 \mathrm{mg}$ of metoprolol daily, which is far less than the maximally tolerated dose, except for case no. 8, who was maintained on a higher dose of carvedilol (25 $\mathrm{mg}$ twice daily) according to a previous long-term prescription. Accordingly, it may be considered that the drop in HR within $48 \mathrm{~h}$ was directly linked to the use of ivabradine rather than $\beta$-blockers. However, the present study was not a prospectively controlled study. Recently, a randomized controlled study compared the efficacy of ivabradine plus $\beta$-blockers versus $\beta$-blockers alone in patients with AHF (11). It suggested that early combined treatment with ivabradine and $\beta$-blockers in congestive HF is feasible and safe. The distinguishing feature of the present study was that all patients were complicated with IRD and had no previous history of HF. The patients in the present study exhibited exacerbated AHF symptoms, and $33.3 \%$ of them underwent mechanical ventilation and did not require $48 \mathrm{~h}$ for stabilization in accordance with data of a previous study (11).

Ivabradine also exhibited sufficient clinical safety in the AHF patients of the present study. No hemodynamic deterioration or impairment of liver or renal function was observed in any of the patients. To the best of our knowledge, no previous study has reported on the application of ivabradine in patients with a 
creatinine clearance of $<15 \mathrm{ml} / \mathrm{min}$. In the present study, case no. 8 had lupus nephritis and started to receive hemodialysis, with an estimated glomerular filtration rate of $9.7 \mathrm{ml} / \mathrm{min}$, due to AHF and reduced urine output. After careful addition of ivabradine, the patient's HF was alleviated and renal perfusion was improved. The patient was void of the requirement for hemodialysis for three months by the end of this study. In addition, according to the manufacturer's guidelines for ivabradine, its use in children and adolescents (age, $<18$ years) is not recommended due to lack of evidence. However, both cases 3 and 4 of the present study were slightly younger than 18 years. After careful communication with their guardians, ivabradine treatment was initiated. The two patients achieved acceptable short-term therapeutic effects using a small dose of ivabradine ( $2.5 \mathrm{mg}$ twice daily) without exhibiting any adverse effects. However, the long-term efficacy and safety of the drug in these patients remains to be determined.

The current study has several limitations. It is a small-sample, single center study without a control group (patients who did not receive ivabradine treatment). Further randomized control studies are therefore required to better testify the efficacy of ivabradine in AHF. Additionally, ivabradine was only evaluated in AHF patients with IRD. It is therefore not clear whether the same treatment could be applied to wider spectrum of patients with AHF.

In conclusion, the present study evaluated early short-term ivabradine treatment in new-onset AHF patients with IRD. The observations suggest that early ivabradine treatment in this type of patients was safe. A significant reduction in HR was observed, and none of the patients developed cardiac shock. Successful control of HR may contribute to hemodynamic and/or heart function improvement, particularly in patients with suspected TCM. Further randomized controlled trials are required to better demonstrate the efficacy and evaluate the long-term prognosis for AHF patients treated with ivabradine.

\section{Acknowledgements}

The authors are grateful for the efforts of Dr Li Zhang, Dr Wei Bai (Department of Rheumatology) and Dr Ke Zheng (Department of Nephrology) from Peking Union Medical College Hospital (Beijing, China) for their special care of the patients.

\section{Funding}

The present study was supported by The National Key Research and Development Program of China (grant nos. 2016YFC0905102 and 2016YFC0901501) and the Chinese Academy of Medical Sciences Innovation Fund for Medical Sciences (grant no. 2017-I2M-2-002).

\section{Availability of data and materials}

The datasets used and/or analyzed during the present study are available from the corresponding author upon reasonable request.

\section{Authors' contributions}

Conception and design: WW and ZS; data collection and/or processing: WW, LZ and YG; materials: JZ, J-JL, DS, JY,
YL and J-ZL; analysis and/or interpretation: YG and ZS; literature search: WW; writing: WW, LZ and ZS; critical review: ZS. All authors confirmed the accuracy of this manuscript.

\section{Ethics approval and consent to participate}

All patients or their legal guardians provided written informed consent. This study complied with the ethical guidelines of the 1964 Declaration of Helsinki, as revised in 2002. The study protocol was reviewed and approved by the ethics committee of Peking Union Medical College Hospital (Beijing, China).

\section{Patient consent for publication}

Not applicable.

\section{Competing interests}

The authors declare that they have no competing interests.

\section{References}

1. Hollan I, Meroni PL, Ahearn JM, Cohen Tervaert JW, Curran S, Goodyear CS, Hestad KA, Kahaleh B, Riggio M, Shields K and Wasko MC: Cardiovascular disease in autoimmune rheumatic diseases. Autoimmun Rev 12: 1004-1015, 2013.

2. Mason JC and Libby P: Cardiovascular disease in patients with chronic inflammation: Mechanisms underlying premature cardiovascular events in rheumatologic conditions. Eur Heart J 36: 482-489c, 2015.

3. Sarzi-Puttini P, Atzeni F, Gerli R, Bartoloni E, Doria A, Barskova T, Matucci-Cerinic M, Sitia S, Tomasoni L and Turiel M: Cardiac involvement in systemic rheumatic diseases: An update. Autoimmun Rev 9: 849-852, 2010.

4. Seferovic PM, Ristic AD, Maksimovic R, Simeunovic DS, Ristic GG, Radovanovic G, Seferović D, Maisch B and Matucci-Cerinic M: Cardiac arrhythmias and conduction disturbances in autoimmune rheumatic diseases. Rheumatology (Oxford) 4 (Suppl 45): iv39-iv42, 2006.

5. Mueller KAL, Heinzmann D, Klingel K, Fallier-Becker P, Kandolf R, Kilias A, Walker-Allgaier B, Borst O, Kumbrink J, Kirchner T, et al: Histopathological and immunological characteristics of tachycardia-induced cardiomyopathy. J Am Coll Cardiol 69: 2160-2172, 2017.

6. Gupta S and Figueredo VM: Tachycardia mediated cardiomyopathy: Pathophysiology, mechanisms, clinical features and management. Int J Cardiol 172: 40-46, 2014.

7. Jeong YH, Choi KJ, Song JM, Hwang ES, Park KM, Nam GB, Kim JJ and Kim YH: Diagnostic approach and treatment strategy cin tachycardia-induced cardiomyopathy. Clin Cardiol 31: $172-178,2008$

8. Thollon C, Cambarrat C, Vian J, Prost JF, Peglion JL and Vilaine JP: Electrophysiological effects of S 16257, a novel sino-atrial node modulator, on rabbit and guinea-pig cardiac preparations: Comparison with UL-FS 49. Br J Pharmacol 112: 37-42, 1994.

9. Bucchi A, Baruscotti M and DiFrancesco D: Current-dependent block of rabbit sino-atrial node I(f) channels by ivabradine. J Gen Physiol 120: 1-13, 2002.

10. Swedberg K, Komajda M, Bohm M, Borer JS, Ford I, Dubost-Brama A, Lerebours G and Tavazzi L; SHIFT Investigators: Ivabradine and outcomes in chronic heart failure (SHIFT): A randomised placebo-controlled study. Lancet 376: 875-885, 2010.

11. Hidalgo FJ, Anguita M, Castillo JC, Rodríguez S, Pardo L, Durán E, Sánchez JJ, Ferreiro C, Pan M, Mesa D, et al: Effect of early treatment with ivabradine combined with beta-blockers versus beta-blockers alone in patients hospitalised with heart failure and reduced left ventricular ejection fraction (ETHIC-AHF): A randomised study. Int J Cardiol 217: 7-11, 2016. 
12. Kim CH, Al-Kindi SG, Jandali B, Askari AD, Zacharias M and Oliveira GH: Incidence and risk of heart failure in systemic lupus erythematosus. Heart 103: 227-233, 2017.

13. Dhakal BP, Kim CH, Al-Kindi SG and Oliveira GH: Heart failure in systemic lupus erythematosus. Trends Cardiovasc Med 28: 187-197, 2018

14. Wolfe $\mathrm{F}$ and Michaud $\mathrm{K}$ : Heart failure in rheumatoid arthritis: Rates, predictors, and the effect of anti-tumor necrosis factor therapy. Am J Med 116: 305-311, 2004.

15. Myasoedova E, Crowson CS, Nicola PJ, Maradit-Kremers H, Davis JM III, Roger VL, Therneau TM and Gabriel SE: The influence of rheumatoid arthritis disease characteristics on heart failure. J Rheumatol 38: 1601-1606, 2011.

16. Mantel A, Holmqvist M, Andersson DC, Lund LH and Askling J: Association between rheumatoid arthritis and risk of ischemic and nonischemic heart failure. J Am Coll Cardiol 69: 1275-1285, 2017.

17. Kyaw H, Misra D, Mani MM, Park WJ and Shinnar M: Unusual cardiac involvement in granulomatosis with polyangiitis manifesting as acute congestive heart failure. Anatol J Cardiol 18: 158-160, 2017.

18. Lundberg IE: The heart in dermatomyositis and polymyositis. Rheumatology (Oxford) 4 (Suppl 45): iv18-iv21, 2006.

19. Kahan A and Allanore Y: Primary myocardial involvement in systemic sclerosis. Rheumatology (Oxford) 4 (Suppl 45): iv14-iv17, 2006.
20. Chen J, Tang Y, Zhu M and Xu A: Heart involvement in systemic lupus erythematosus: A systemic review and meta-analysis. Clin Rheumatol 35: 2437-2448, 2016.

21. Wang CR, Tsai YS and Li WT: Lupus myocarditis receiving the rituximab therapy-a monocentric retrospective study. Clin Rheumatol 37: 1701-1707, 2018.

22. Pascual Izco M, Alonso Salinas GL, Sanmartin Fernandez M, Del Castillo Carnevalli H, Jimenez Mena M, Camino Lopez A and Zamorano Gómez JL: Clinical experience with ivabradine in acute heart failure. Cardiology 134: 372-374, 2016.

23. Fox K, Ford I, Steg PG, Tendera M, Robertson M and Ferrari R; BEAUTIFUL Investigators: Relationship between ivabradine treatment and cardiovascular outcomes in patients with stable coronary artery disease and left ventricular systolic dysfunction with limiting angina: A subgroup analysis of the randomized, controlled BEAUTIFUL trial. Eur Heart J 30: 2337-2345, 2009.

24. Fox K, Ford I, Steg PG, Tardif JC, Tendera M and Ferrari R; SIGNIFY Investigators: Ivabradine in stable coronary artery disease without clinical heart failure. N Engl J Med 371: 1091-1099, 2014.

25. Tardif JC,Ponikowski P and Kahan T; ASSOCIATE Investigators: Effects of ivabradine in patients with stable angina receiving beta-blockers according to baseline heart rate: An analysis of the ASSOCIATE study. Int J Cardiol 168: 789-794, 2013. 